\title{
Utilisation d'une étude cas-témoins et d'une banque de témoins afin de mener une enquête sur une éclosion de cyclosporose d'origine locale au Canada en 2016
}

\begin{abstract}
V Morton ${ }^{1 *}$, K Meghnath², M Gheorghe ${ }^{2}$, A Fitzgerald-Husek ${ }^{3}$, J Hobbs ${ }^{4}$, L
\section{Résumé}

Contexte : Cyclospora est un parasite intestinal qui n'est pas endémique au Canada. Toutefois, des éclosions nationales de cas d'infection contractée à l'échelle locale ont été signalées depuis 2013.

Ces éclosions étaient probablement associées à la consommation de produits importés de pays où Cyclospora est endémique. L'identification de la source peut s'avérer difficile en raison des délais de signalements et de la traçabilité limitée des produits.
\end{abstract}

Objectif : Rapporter une éclosion nationale de cyclosporose d'origine locale, faire ressortir les défis d'enquêter sur ces éclosions, et documenter l'utilisation d'une banque de témoins pour la première fois en vue de recruter des témoins dans le cadre d'une étude d'éclosion cas-témoin nationale au Canada.

Méthodologie : Les cas de cyclosporose ont été identifiés par le biais de tests de laboratoire provinciaux et signalés par les provinces au niveau national. Les cas ont été interrogés sur les expositions alimentaires au moyen d'un questionnaire, et les expositions alimentaires rapportées par les cas ont été comparées aux valeurs de référence du Foodbook. Pour circonscrire les produits alimentaires d'intérêt, une étude cas-témoins avec appariement a été réalisée. Les groupes témoins de l'étude ont été recrutés principalement à partir d'une banque de témoins, c'est-à-dire une liste de personnes qui avaient précédemment accepté de participer à des enquêtes relatives à la santé publique.

Résultats : Au total, 87 cas de cyclosporose d'origine locale, dont les dates d'apparition ou de déclaration se situent entre le 19 mai et le 10 août 2016, ont été signalés par quatre provinces. Comparer les cas d'exposition aux valeurs de référence Foodbook a permis de déterminer plusieurs produits alimentaires d'intérêt, notamment les mûres, d'autres baies, des fines herbes et des légumes feuillus. L'étude cas-témoins indique que les mûres et les salades mesclun ont plus été consommées par les cas que par les témoins. En raison du manque de détails sur les produits pour les mûres et le mesclun, la source de l'épidémie n'a pas été établie de façon concluante.

Conclusion : Les mûres constituaient le principal produit alimentaire d'intérêt, mais elles n'ont pas pu être établies comme source concluante en raison du manque de traçabilité. La banque de témoins s'est révélée être un outil utile pour le recrutement.

Citation proposée : Morton V, Meghnath K, Gheorghe M, Fitzgerald-Husek A, Hobbs J, Honish L., David S. Utilisation d'une étude cas-témoins et d'une banque de témoins afin de mener une enquête sur une éclosion de cyclosporose d'origine locale au Canada en 2016. Relevé des maladies transmissibles au Canada 2019;45(9):

247-52. https://doi.org/10.14745/ccdr.v45i09a01f

Mots-clés : Cyclospora, éclosion, étude cas-témoins, cyclosporose, Canada
Cette oeuvre est mise à la disposition selon les termes de la licence internationale Creative Commons Attribution 4.0

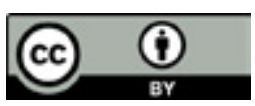

Affiliations

${ }^{1}$ Agence de la santé publique du Canada, Guelph, Ontario

${ }^{2}$ Agence de la santé publique du Canada, Ottawa, Ontario

${ }^{3}$ Service de santé publique de la région de York, Newmarket, Ontario

${ }^{4}$ Santé publique Ontario, Toronto, Ontario

${ }^{5}$ Services de santé de l'Alberta, Edmonton, Alberta

${ }^{6}$ Centre de contrôle des maladies de la Colombie-Britannique, Vancouver, Colombie-Britannique

\section{*Correspondance:}

vanessa.morton@canada.ca 


\section{Introduction}

Cyclospora cayetanensis est un parasite entérique qui cause la gastroentérite (1). Cyclospora n'est pas endémique au Canada; les cas sont souvent associés à des voyages internationaux. Les cas de cyclosporose d'origine locale sont probablement associés à la consommation de produits importés de pays où Cyclospora est endémique (2). Par exemple, dans les années 90, plusieurs éclosions ont été associées à des framboises importées du Guatemala; des restrictions à l'importation ont mis fin à ces éclosions (3). Depuis 2013, des éclosions nationales de cyclosporose d'origine locale se produisent au Canada chaque printemps et durant l'été. Cependant, l'identification de la source a été difficile en raison du manque de méthode de typage moléculaire, des retards considérables dans le signalement des cas et de la traçabilité limitée des produits.

Une étape importante dans les enquêtes sur les éclosions $d$ 'origine alimentaire est la génération d'hypothèses, et l'une des techniques de génération d'hypothèses consiste à comparer les expositions alimentaires signalées par les cas aux expositions rapportées par la population générale (4). Au Canada, les enquêteurs peuvent utiliser les valeurs de référence du Foodbook pour les comparaisons. Le Foodbook est le résultat d'un sondage téléphonique effectué auprès de la population, qui a été mené dans toutes les provinces et tous les territoires du Canada pendant un an (en 2014 à 2015), et dont le principal objectif était de décrire les aliments consommés par les Canadiens sur une période de sept jours. Les études castémoins sont également couramment utilisées pour la génération d'hypothèses lors d'enquêtes sur les éclosions. Cependant, elles peuvent être longues et coûteuses à mener. L'un des défis associés à ces études est de disposer d'une méthode efficace pour recruter des témoins. Une banque de témoins a été créée dans le cadre de l'étude Foodbook afin de faire face à ce défi. On a demandé aux participants s'ils souhaitaient être contactés pour prendre part à d'autres enquêtes liées à des éclosions ou projets de recherche. L'utilisation d'une banque de témoins pour recruter des participants a été une réussite en Australie, particulièrement en ce qui concerne les enquêtes sur les éclosions et d'autres projets de recherche en santé publique dans ce pays $(5,6)$.

L'objectif de ce rapport est de décrire l'enquête portant sur une éclosion à l'échelle nationale de cyclosporose d'origine locale en 2016, et de mettre en lumière certains des défis associés à l'enquête sur cette éclosion au Canada.

\section{Détection des éclosions}

Le 27 juin 2016, des responsables de la santé publique de la Colombie-Britannique ont informé l'Agence de la santé publique du Canada (ASPC) de leur premier cas de cyclosporose d'origine locale cette année-là. En date du 9 juillet 2016, l'Ontario et l'Alberta avaient signalé huit cas d'origine locale. L'ASPC, en collaboration avec des partenaires locaux, provinciaux et fédéraux, a rapidement lancé une enquête nationale sur ces éclosions.

\section{Méthodologie}

\section{Enquête épidémiologique}

Un cas associé à l'éclosion de cyclosporose fut défini comme étant une infection confirmée en laboratoire par $C$. cayetanensis chez un résident ou un visiteur du Canada, avec l'apparition des symptômes le $1^{\text {er }}$ avril 2016 ou après cette date, et sans antécédent de voyage à l'extérieur du Canada ou des États-Unis au cours des 14 jours précédant l'apparition des symptômes. Les cas ont été dépistés conformément aux normes de diagnostic provinciales et signalés à l'ASPC par les responsables provinciaux de la santé publique. Des données relatives aux expositions alimentaires au cours des 14 jours précédant l'apparition de la maladie ont été obtenues à l'aide d'un questionnaire spécifique à la cyclosporose. Certains cas ont été interrogés à nouveau par des enquêteurs centralisés au niveau national ou local si les entretiens initiaux n'avaient pas permis d'obtenir les détails requis sur les expositions ou le produit. Des données ont été recueillies sur l'exposition aux baies fraîches, aux herbes, aux légumes feuillus, aux pois et à d'autres légumes; seule l'exposition aux produits frais a été prise en compte. Les fréquences d'exposition ont été comparées aux valeurs de référence des aliments de l'étude Foodbook pour les mois de mai, juin, juillet et août pour les provinces touchées, en utilisant des probabilités binomiales et un seuil de signification de $p=0,05(7,8)$.

\section{Étude cas-témoins}

Une étude cas témoins a été menée pour déterminer les aliments qui sont associés à la maladie. Les cas pouvaient être inclus dans l'étude s'ils répondaient à la définition de cas d'éclosion et avaient à la fois un questionnaire raisonnablement complet ainsi qu'une date connue d'apparition de la maladie. La date d'apparition devait être après le 31 mai 2016, et la maladie devait avoir été signalée au plus tard le 26 septembre 2016. Les témoins ont été jumelés à chaque cas en fonction du groupe d'âge (10 à 19 ans, 20 à 69 ans et plus de 70 ans) ainsi que leur lieu de résidence en se basant sur le premier chiffre du code postal (9). Les témoins ont été interrogés sur leurs expositions alimentaires pendant la même période de 14 jours que les cas. Trois témoins ont été sélectionnés pour chaque cas afin de maximiser la puissance statistique des résultats en fonction de la taille estimée de l'échantillon. Le recrutement s'est déroulé à l'aide d'une banque de témoins, c'est-à-dire un répertoire des coordonnées de personnes interrogées dans le cadre de l'étude Foodbook, qui avaient accepté d'être contactées à des fins de recherche ou d'enquête. Le recrutement supplémentaire, une fois la banque de témoins épuisée, a été effectué via la méthode de composition aléatoire. 
Les données des cas-témoins ont été analysées à l'aide des rapports de cotes $(R C)$ de McNemar pour les cas appariées. Les facteurs relevés dans l'analyse à une variable, avec des RC supérieurs à un et une valeur de $p$ inférieure à 0,2 , ont été inclus dans un modèle de régression multivariable. Les facteurs ont été retirés en utilisant une régression progressive descendante; les variables demeurent dans le modèle si elles modifient les coefficients de signification par plus de $20 \%(10,11)$.

\section{Résultats}

\section{Enquête épidémiologique}

Au total, 87 cas de cyclosporose d'origine locale ont été relevés dans quatre provinces (Ontario, $n=75$; Québec, $n=8$; Alberta, $n=2$; Colombie-Britannique, $n=2$ ). Les dates de l'apparition des symptômes et de prélèvement de l'échantillon s'échelonnaient du 19 mai au 10 août 2016 (figure 1). Un cas a été exclu de l'historique alimentaire en raison de l'incertitude entourant la date du début (signalée à la « mi-juillet ») et du long délai écoulé entre la période d'exposition et l'entretien (la date d'isolement était le 23 septembre 2016).

Figure 1 : Nombre de cas confirmés d'éclosion de cyclosporose d'origine locale, par date d'apparition des symptômes ou de prélèvement de l'échantillon, et statut d'inclusion ou d'exclusion dans l'étude castémoins, 2016, Canada ${ }^{a}$

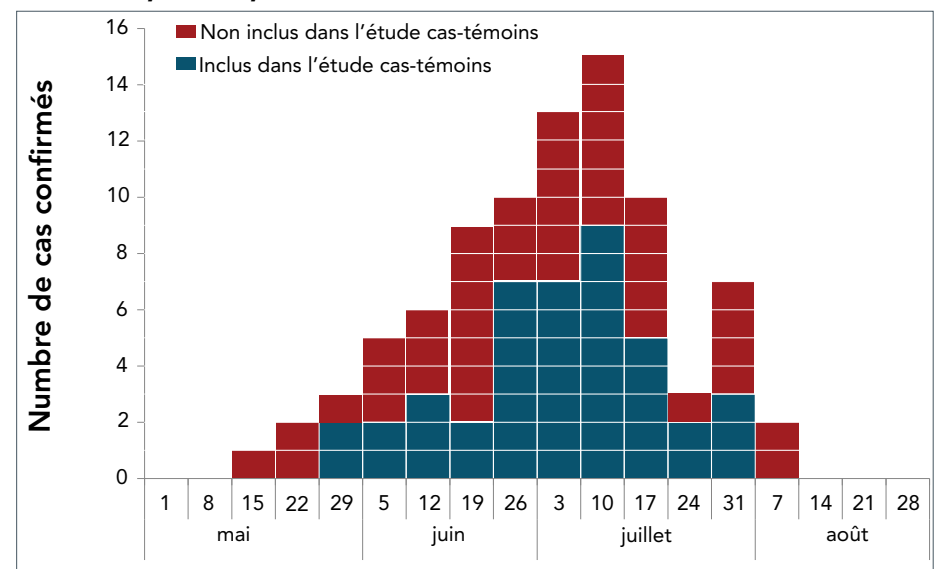

Semaine de l'apparition des symptômes ou du prélèvement de l'échantillon $(n=86)$

a Un cas d'éclosion a été exclu, car aucune date n'était disponible relativement à l'apparition des symptômes ni au prélèvement de l'échantillon; seule une date d'isolement était disponible

L'âge médian des cas était de 51 ans (intervalle de 15 à 89 ans) et $52 \%$ étaient des femmes. Un cas a été hospitalisé. II n'y a eu aucun décès.

Les données relatives à l'exposition étaient disponibles pour 86 cas. Les produits alimentaires les plus fréquemment signalés étaient la laitue romaine $(51 / 70,73 \%)$, les fraises $(51 / 74,69 \%)$ et les autres baies $(51 / 74,69 \%)$ (tableau 1). Comparativement aux valeurs de référence du Foodbook, les cas de cyclosporose ont signalé les sept expositions alimentaires suivantes beaucoup plus fréquemment $(p \geq 0,05)$ que la population générale : framboises, mûres, coriandre, persil, laitue romaine, épinards et mesclun.

\section{Étude cas-témoins}

Quarante-deux cas répondaient aux critères d'inclusion de l'étude cas-témoin. Ils ont été appariés avec 126 témoins (117 de la banque de témoins et neuf inscrits via la méthode de composition aléatoire). Les taux de réponse pour la banque de témoins et la méthode de composition aléatoire ont été respectivement de $60 \%$ et de $24 \%$.

Une analyse appariée univariée a indiqué que les cas étaient beaucoup plus susceptibles que les témoins d'avoir été exposés à des mûres $(\mathrm{RC}=2,50$; intervalles de confiance $[\mathrm{IC}]$ à $95 \%=1,16$ à 4,91) et au mesclun $(R C=2,50$; IC à $95 \%=1,01-$ 5,48 ) (tableau 1). Une analyse multivariée utilisant une méthode de régression progressive descendante a révélé que seule l'exposition aux mûres était significative; l'exposition au mesclun et à la roquette a été incluse dans le modèle à titre de variable confusionnelle.

\section{Enquête sur la salubrité des aliments}

À partir des résultats de l'étude cas-témoins, des tentatives ont été effectuées pour repérer les aliments qui étaient beaucoup plus susceptibles d'avoir été consommés par les cas. Les renseignements sur la marque, les dates d'achat, les cartes de fidélité et les enregistrements des commerces ont été utilisés. Des données sur les importations ont été demandées en ce qui concerne les mûres et le mesclun pour la période de mai à août 2016; les données relatives à l'importation concernant les salades mesclun n'étaient pas disponibles.

Un même pays d'origine ou un fournisseur commun de mûres n'a pas été identifié compte tenu du peu de renseignements disponibles. Le Mexique était le plus gros fournisseur de mûres en mai, représentant $64 \%$ des importations canadiennes. Cette part de marché a diminué à $20 \%$ en juin et de $3 \%$ à $4 \%$ en juillet et août. Pendant ces deux mois, la majorité des mûres importées au Canada provenaient des États-Unis. Le Guatemala était à l'origine de $0,2 \%$ des importations de mûres de mai à août.

\section{Intervention de santé publique}

Le public a été informé de la flambée de cas de cyclosporose $d^{\prime}$ origine locale par le biais d'un avis de santé publique affiché sur le site Web de I'ASPC. Puisqu'aucun produit spécifique n'a été repéré, des recommandations générales de prévention ont été fournies. Ces recommandations incitent les gens à cuire les produits importés de pays où Cyclospora est présent, et à consommer des produits frais cultivés dans des pays où Cyclospora n'est pas courant. On a conseillé aux voyageurs se rendant dans un pays où Cyclospora est présent d'éviter les aliments lavés dans de l'eau potable locale, de ne boire que de l'eau provenant d'une source sûre, et de manger des aliments cuits ou des fruits pouvant être pelés. 
Tableau 1 : Résultats de la comparaison de probabilité binomiale entre les valeurs de référence du Foodbook et les rapports de cotes de l'étude cas-témoins

\begin{tabular}{|c|c|c|c|c|c|c|c|}
\hline \multirow[b]{2}{*}{$\begin{array}{l}\text { Produit } \\
\text { alimentaire }\end{array}$} & \multicolumn{3}{|c|}{ Référence Foodbook (rappel de sept jours) ${ }^{\mathrm{a}}$} & \multicolumn{4}{|c|}{ Étude cas-témoins (rappel de 14 jours) ${ }^{\mathrm{b}}$} \\
\hline & $\begin{array}{c}\text { Cas } \\
\text { exposés (\%) }\end{array}$ & $\begin{array}{c}\text { Témoins }{ }^{c} \\
\text { exposés (\%) }\end{array}$ & $\begin{array}{l}\text { Probabilité } \\
\text { binomiale } \\
\text { (Valeur de p) }\end{array}$ & Cas exposés (\%) & $\begin{array}{c}\text { Témoins } \\
\text { exposés (\%) }\end{array}$ & $\begin{array}{l}\text { Rapport des } \\
\text { cotes }\end{array}$ & Valeur de $p$ \\
\hline Framboises & 69 & 64 & 0,007 & 68 & 79 & 0,57 & 0,16 \\
\hline Mûres & 51 & 30 & $<0,001$ & 53 & 50 & 1,09 & 0,81 \\
\hline Autres baies & 43 & 11 & $<0,001$ & 49 & 28 & 2,50 & 0,02 \\
\hline Basilic & 69 & $\begin{array}{l}\text { Aucune } \\
\text { donnée }^{d}\end{array}$ & S. O. & 73 & 59 & 1,86 & 0,12 \\
\hline Coriandre & 23 & 22 & 0,11 & 24 & 35 & 0,60 & 0,23 \\
\hline Persil & 42 & 19 & $<0,001$ & 38 & 39 & 0,96 & 0,93 \\
\hline $\begin{array}{l}\text { Autres herbes } \\
\text { fraîches }\end{array}$ & 47 & 33 & 0,01 & 53 & 39 & 1,78 & 0,16 \\
\hline Laitue Iceberg & 27 & $\begin{array}{l}\text { Aucune } \\
\text { donnée }^{\text {d }}\end{array}$ & S. O. & 32 & 24 & 1,48 & 0,37 \\
\hline Laitue romaine & 39 & 44 & 0,06 & 44 & 64 & 0,45 & 0,03 \\
\hline Épinards & 73 & 53 & $<0,001$ & 76 & 84 & 0,60 & 0,27 \\
\hline $\begin{array}{l}\text { Mélange de } \\
\text { salade mesclun }\end{array}$ & 48 & 33 & 0,003 & 50 & 67 & 0,49 & 0,06 \\
\hline Roquette & 30 & 18 & 0,003 & 30 & 15 & 2,50 & 0,03 \\
\hline $\begin{array}{l}\text { Autres laitues ou } \\
\text { légumes-feuilles }\end{array}$ & 42 & $\begin{array}{l}\text { Aucune } \\
\text { donnée }^{\text {d }}\end{array}$ & S. O. & 46 & 31 & 1,92 & 0,13 \\
\hline Pois & 43 & $\begin{array}{l}\text { Aucune } \\
\text { donnée }^{d}\end{array}$ & S. O. & 46 & 42 & 1,18 & 0,66 \\
\hline Pois mange-tout & 15 & 29 & 0,002 & 20 & 37 & 0,42 & 0,05 \\
\hline Pois sucrés & 10 & $\begin{array}{l}\text { Aucune } \\
\text { donnée }^{d}\end{array}$ & S. O. & 11 & 26 & 0,35 & 0,17 \\
\hline
\end{tabular}

Abréviations : S. O., sans objet; <, inférieur à

a La référence Foodbook était basée sur tous les cas $(n=86)$

'L'étude cas-témoins était basée sur les cas éligibles $(n=42)$ et les témoins $(n=126)$

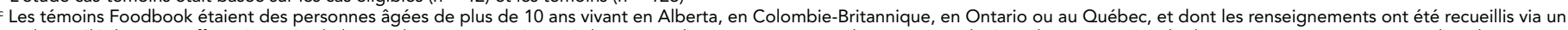
sondage téléphonique effectué auprès de la population, qui a été mené dans toutes les provinces et tous les territoires du Canada sur une période d'un an (2014 et 2015); ceci incluait les gens interviewés entre mai et septembre 2016 (étude non publiée)

dÉtant donné que ces produits alimentaires n'étaient pas inclus dans l'enquête Foodbook, la probabilité binomiale n'a pas été calculée

\section{Discussion}

Cette enquête sur les éclosions met en relief certains des problèmes liés à l'identification de la source des éclosions de cyclosporose. Malgré une enquête approfondie, la source de cette éclosion n'a pas été établie. Toutefois, les renseignements sur l'exposition provenant du Foodbook et l'étude cas-témoins ont été utiles pour formuler des hypothèses. À notre connaissance, cette éclosion a donné lieu à la première occasion de mettre sur pied une étude cas-témoins, menée dans le cadre d'une enquête sur une éclosion nationale de cyclosporose au Canada. De plus, la banque de témoins s'est révélée un outil efficace pour le recrutement de témoins.

Bien que les mûres n'aient pas été identifiées de façon concluante comme étant la source de l'éclosion, elles ont été ciblées comme un produit alimentaire d'intérêt à la fois dans le Foodbook et dans l'étude cas-témoins. En outre, l'étude cas-témoins a permis de renforcer la preuve que les mûres constituaient une source suspecte. La seule raison pour laquelle ces résultats ne pouvaient pas prouver que les mûres étaient à l'origine de l'éclosion était le manque de précision quant à la marque ou le producteur de mûres consommées par les cas. Ces résultats concordaient avec les éclosions de cyclosporose d'origine locale contractées au Canada en 2014 et 2015, alors que les mûres avaient été signalées comme étant l'une des multiples sources présumées de la flambée (Agence de la santé publique du Canada. Enquête multiprovinciale sur les éclosions de cyclosporose en Colombie-Britannique, au Québec et en Ontario : Résumé épidémiologique définitif, Rapport non publié). Cette conclusion est également corroborée par l'examen des données d'importation de mûres, qui a révélé que le Mexique était un important fournisseur de mûres au Canada en mai et au début de juin. Compte tenu de la période d'incubation de la cyclosporose (jusqu'à 14 jours) et de la durée de conservation des mûres (jusqu'à 21 jours), les maladies associées à ces mûres auraient pu survenir plus d'un mois après l'importation, concordant avec le moment le plus fort de l'éclosion, au mois de juillet $(3,12)$. Cyclospora est endémique au Mexique et des cas 
de cyclosporose ont été signalés chez des Canadiens voyageant au Mexique (13). Il est vraisemblable qu'un autre produit ou plusieurs produits alimentaires aient également contribué à cette éclosion, puisque seulement $43 \%$ des cas ont signalé avoir consommé des mûres; de plus, le pourcentage de cas déclarant avoir consommé des mûres diminue parmi ceux ayant signalé des dates d'apparition tardive de la maladie.

La comparaison du Foodbook et l'analyse cas-témoins ont toutes deux permis de déterminer que le mesclun constituait un autre sujet d'intérêt. Cependant, l'interprétation de cette constatation a été restreinte par le manque d'uniformité relativement aux types de laitues identifiés comme des " salades mesclun » et par un manque de détails concernant l'emballage et la marque. Ces motifs ont empêché de déterminer de façon concluante l'identification d'un produit spécifique.

\section{Forces et limites}

Cette éclosion a démontré l'utilité d'une banque de témoins pour faciliter le recrutement dans le cadre d'une étude castémoins. Le fait de posséder une liste de personnes disposées à participer à des enquêtes a facilité l'amorce rapide et rentable d'une étude cas-témoins. En outre, le taux de réponse de la banque de témoins a été beaucoup plus élevé que celui de la méthode de composition aléatoire. La baisse de l'usage des téléphones filaires par les ménages, en plus de la grande disponibilité de l'afficheur, ont compliqué le recrutement de personnes par l'entremise de la méthode de composition aléatoire. Cependant, I'utilisation d'une banque de témoins introduit la possibilité d'un biais de sélection, car les personnes qui acceptent de participer à la banque de témoins peuvent être fort différentes de la population en général.

Une autre limite importante à considérer est que les périodes de temps relatives à l'historique alimentaire pour les données de l'étude de cas-témoins et les données du Foodbook différaient : les participants au Foodbook ont été interrogés sur un historique alimentaire de sept jours, alors que les cas de cette enquête ont été interrogés sur les expositions pendant 14 jours, en se fondant sur la période d'incubation de Cyclospora. Cette différence quant à la période de rappel peut avoir eu comme conséquence que davantage de produits alimentaires ont été signalés, et ce, à des fréquences plus élevées, par les cas plutôt que par la population de référence de Foodbook.

\section{Prochaines étapes}

Les études cas-témoins constituent un outil d'investigation utile, car elles permettent de déceler une source alimentaire plus susceptible d'être consommée par les cas liés aux éclosions que par les témoins; cependant, des données de laboratoire et/ou des renseignements de traçabilité supplémentaires sont nécessaires pour désigner la source avec certitude.

Cette enquête met également en évidence la nécessité de revoir les stratégies visant à assurer l'efficacité des enquêtes liées à la cyclosporose d'origine locale. La collecte de données de qualité relativement à l'achat et à l'exposition aux aliments par les cas au moment de l'entretien initial est essentielle, mais difficile, en raison de la longue période d'incubation et de la durée de conservation limitée des produits. L'absence de méthodes de laboratoire couramment disponibles pour typer Cyclospora constitue un autre enjeu majeur en matière d'investigation. Une solution envisageable serait de concentrer les efforts sur le dépistage et l'examen de grappes ou agrégats d'événements ou de lieux où les cas sont plus susceptibles d'avoir contracté la maladie après avoir été en contact avec un produit alimentaire contaminé. Des discussions plus poussées avec les pays exportateurs de produits où Cyclospora est endémique pourraient aider à mettre en place des mesures de prévention et de contrôle.

\section{Conclusion}

Les résultats de cette enquête sur les éclosions nous permettent de mieux comprendre cette maladie au Canada, en plus de démontrer qu'une banque de témoins peut constituer un outil efficace en vue de mener des études cas-témoins dans le cadre d'enquêtes de santé publique.

\section{Déclaration des auteurs}

V. M. - Enquête, méthodologie, rédaction (première ébauche, examen et révision)

K. M. et M. G. - Enquête, méthodologie, rédaction (examen et révision)

A. F. H., J. H., L. H. et S. D. - Enquête, rédaction (examen et révision)

\section{Conflit d'intérêts}

Aucun.

\section{Remerciements}

Nous tenons à remercier nos collègues du Centre de lutte contre la maladie de la Colombie-Britannique, de Santé Alberta, des Services de santé de l'Alberta, de Santé publique Ontario, du ministère de la Santé et des soins de longue durée de l'Ontario, du ministère de la Santé et des services sociaux du Québec, de l'Agence canadienne d'inspection des aliments, de Santé Canada et de l'Agence de la santé publique du Canada, ainsi que des laboratoires de santé publique en Colombie-Britannique, en Alberta, en Ontario et au Québec.

\section{Financement}

Ce travail a été réalisé grâce au soutien de l'Agence de la santé publique du Canada. 
7. Centre des Maladies Infectieuses d'origine Alimentaire, Environnementale et Zoonotique, Direction Générale de la Prévention et Contrôle des Maladies Infectieuses, Agence de la Santé Publique du Canada. Rapport foodbook. Guelph (ON): Agence de la Santé Publique du Canada; 2015. http:// publications.gc.ca/collections/collection_2016/aspc-phac/ HP40-146-2015-fra.pdf

2. Hedberg CW, Osterholm MT. Foodborne outbreaks caused by Cyclospora: the message is more important than the messenger. Epidemiol Infect 2016 Jul;144(9):1803-6. DOI PubMed

3. Herwaldt BL, Beach MJ; Cyclospora Working Group. The return of Cyclospora in 1997: another outbreak of cyclosporiasis in North America associated with imported raspberries. Ann Intern Med 1999 Feb;130(3):210-20. DOI PubMed

4. CIFOR. Guidelines for foodborne disease outbreak response (CIFOR Guidelines). Atlanta (GA): Council to Improve Foodborne Outbreak Response; 2009. https://cifor.us/ products/guidelines

5. Munnoch SA, Ward K, Sheridan S, Fitzsimmons GJ, Shadbolt CT, Piispanen JP, Wang Q, Ward TJ, Worgan TL, Oxenford C, Musto JA, McAnulty J, Durrheim DN. A multi-state outbreak of Salmonella Saintpaul in Australia associated with cantaloupe consumption. Epidemiol Infect 2009 Mar;137(3):367-74. DOI PubMed

6. Stafford RJ, Schluter P, Kirk M, Wilson A, Unicomb L, Ashbolt R, Gregory J and the OzFoodNet Working Group. A multi-centre prospective case-control study of campylobac-ter infection in persons aged 5 years and older in Australia. Epidemiol Infect 2007 Aug;135(6):978-88. DOI PubMed

8. Gaulin C, Levac E, Ramsay D, Dion R, Ismaïl J, Gingras S, Lacroix C. Escherichia coli O157:H7 outbreak linked to raw milk cheese in Quebec, Canada: use of exact probability calculation and casecase study approaches to foodborne outbreak investigation. J Food Prot 2012 May;75(5):812-8. DOI PubMed

9. Code postal : Définition détaillée. Ottawa (ON): Statistique Canada; (Accédé 2018-09-17). https://www150.statcan. gc.ca/n1/pub/92-195-x/2011001/other-autre/pc-cp/pc-cpfra.htm

10. Rimhanen-Finne R, Niskanen T, Hallanvuo S, Makary $P$, Haukka K, Pajunen S, Siitonen A, Ristolainen R, Pöyry H, Ollgren J, Kuusi M. Yersinia pseudotuberculosis causing a large outbreak associated with carrots in Finland, 2006. Epidemiol Infect 2009 Mar;137(3):342-7. DOI PubMed

11. Dohoo I, Martin W, Stryhn H, editors. Methods in epidemiologic research. Charlottetown (PEI): VER Inc.; 2012.

12. Perkins-Veazie P, Collins JK, Clark JR. Shelf-life and quality of 'Navaho' and 'Shawnee' blackberry fruit stored under retail storage conditions. J Food Qual 1999;22(5):535-44. DOI

13. Nichols GL, Freedman J, Pollock KG, Rumble C, Chalmers RM, Chiodini P, Hawkins G, Alexander CL, Godbole G, Williams C, Kirkbride HA, Hamel M, Hawker JI. Cyclospora infection linked to travel to Mexico, June to September 2015. Euro Surveill 2015;20(43): DOI PubMed 